\title{
Le signal Notch, CADASIL et syndrome d'Alagille
}

Le signal Notch joue un rôle décisif dans la différenciation, et probablement dans la prolifération de nombreux lignages cellulaires. Mais ce n'est que tout récemment qu'on a pris conscience de son importance chez l'homme $\left(\mathrm{m} / \mathrm{s} n^{\circ} 6-7\right.$, vol. 13, p. 914). La voie Notch était surtout connue des drosophilistes qui décrivirent le premier locus il y a près de 80 ans: la perte d'une copie du gène Notch confère à la drosophile femelle un phénotype à ailes encochées (notched wings) (à l'origine de son nom), mais surtout, avec les ligands Delta et Serrate, ce gène règle la différenciation des cellules précurseurs du système nerveux [1]. Chez Caenorhabditis elegans, on trouve d'autres ligands Lag-2 et APX-1. Enfin, chez les vertébrés, il existe plusieurs gènes Notch (probablement par duplication du gène initial au cours de l'évolution) et différents ligands dont le catalogue complet est encore en cours d'établissement. Ces protéines transmembranaires sont constituées, entre autres, d'une structure extramembranaire pouvant posséder une région riche en cystéine, une série variable de répétition EGF (epithelial growth factor) et, dans la région amino-terminale, d'un domaine qui comprend environ 40 acides aminés, appelé DSL parce que commun à Delta, Serrate et Lag-2.

De même que, chez la souris, des mutations de Notch4, avec insertion virale, sont responsables de tumeurs, les quatre gènes $N O T C H$ isolés chez l'homme sont tous localisés en des points de translocations associées à des processus néoplasiques [2]. On s'attendait à les trouver en cause en oncologie plutôt que dans des maladies génétiques humaines constitu- tionnelles transmises en dominance, surtout à début tardif. Pourtant on est désormais certain que le gène NOTCH3 est impliqué dans CADASIL et que le ligand du récepteur Notch1, JAG1, est muté dans le syndrome d'Alagille.

CADASIL (pour autosomal dominant arteriopathy with subcortical infarcts and leucoencephalopathy) est une des causes de démence vasculaire, les démences vasculaires représentant la deuxième cause de démence après la maladie d'Alzheimer. Les caractéristiques cliniques et génétiques de cette maladie ont été établies lors de l'étude d'une grande famille française grâce à une étroite collaboration entre le groupe de neurologie dirigé par le Pr M.G. Bousser (CHU Lariboisière) et l'équipe de génétique du Dr E. Tournier-Lasserve [3]. Cette affection est caractérisée par la survenue d'infarctus cérébraux récidivants et la présence de lésions diffuses de la substance blanche cérébrale, détectables en IRM (imagerie par résonance magnétique) avant même l'apparition des symptômes. Une autre des caractéristiques de cette affection est la présence d'un matériel granulaire osmiophile bien visible au microscope électronique dans la membrane basale des cellules musculaires lisses des parois vasculaires, non seulement dans les vaisseaux cérébraux mais aussi dans les artérioles cutanées [4]. Une analyse de liaison génétique conduite sur cette première famille par l'équipe du Dr Tournier-Lasserve avait permis en 1993 de localiser le gène responsable sur le chromosome 19 [5].

Grâce à l'analyse de nouvelles familles, cette équipe réussit à limiter l'intervalle critique à $800 \mathrm{~kb}$ environ.
Par sélection d'ADNc, de nombreux transcrits furent identifiés. L'un d'eux présentait dans sa région 5' codante une forte similitude avec le gène Notch 3 de la souris. La constatation de la grande ressemblance entre Sel12 (gène intervenant dans la voie de signalisation Notch chez C. elegans) et le gène codant pour la préséniline 1 chez l'homme $(\mathrm{m} / \mathrm{s}$ n०8-9, vol. 13, p. 1036) a conduit cette équipe à émettre l'hypothèse selon laquelle la voie de signalisation Notch pouvait être impliquée dans cette démence de l'adulte [6].

Après avoir isolé et ordonné d'autres segments d'ADNc, une bande de 5615 paires de bases de la séquence codante du gène NOTCH3 humain fut assemblée [6]. Les protéines déduites, murines et humaines, sont identiques à $90 \%$. Aucune lésion grossière du gène n'ayant été observée chez des malades atteints de CADASIL, le groupe entreprit donc de rechercher des mutations ponctuelles en criblant 14 des 29 exons connus de la séquence génomique. Sur 14 malades non apparentés, dix mutations furent découvertes qui coségrègent avec la maladie dans les familles étudiées. Neuf mutations sont situées dans sept différents domaines EGF, la dixième se trouve dans un domaine de répétitions ankyrin-like $(c d c-10)$, et toutes touchant des acides aminés très conservés semblent devoir entraîner la production d'une protéine gravement désorganisée. Toutefois, aucune différence phénotypique ne put être distinguée selon les diverses mutations et on attend avec impatience de savoir par quel mécanisme pathogénique les mutations du gène NOTCH3 sont responsables de cette 
leucoencéphalopathie vasculaire progressive.

Le syndrome d'Alagille est, lui aussi, transmis en dominance mais il se manifeste dès les premiers jours de vie par l'apparition d'un ictère dû à une cholestase par insuffisance des voies biliaires. Toutefois, il existe des formes frustes, souvent découvertes lors de l'examen de parents de nourrissons touchés par une forme majeure de cette dysplasie artériohépatique. Le syndrome comporte, en outre, de très nombreuses autres anomalies: malformations vasculaires, vertébrales, oculaires, ainsi qu'une dysmorphie faciale caractéristique: front large, menton pointu, extrémité du nez bulbeuse, cavités orbitaires profondes.

La localisation du gène en 20p12 fut facilitée par des délétions interstitielles observées chez certains malades $\left(\mathrm{m} / \mathrm{s} n^{\circ} 1\right.$, vol. 7, p. 187) [7]. Après construction d'un contig de BAC (bacterial artificial chromosome), l'analyse de la plus petite région critique révéla la présence du locus d'un des ligands du récepteur Notch1, JAG1, homologue du gène murin Jagged 1 , connu pour activer Notch1 et inhiber la différenciation des cellules musculaires en culture [8]. Chez la souris, pendant le développement embryonnaire, on détecte l'expression du gène dans les deux premiers arcs branchiaux, la vésicule optique, la crête neurale, le cerveau, le cour et les bourgeons de la queue et des pattes postérieures. Encore fallait-il confirmer l'implication de $J A G 1$ dans tous les syndromes d'Alagille, en particulier chez les malades (les plus nombreux) chez lesquels aucune microdélétion n'avait pu être détectée [9]. Les deux équipes américaines $[8,10]$ ayant isolé ce gène de 26 exons dont l'ARNm mesure $5,5 \mathrm{~kb}$ environ, vien- nent effectivement de mettre en évidence plusieurs mutations intragéniques (délétions d'une ou deux paires de bases, insertions, mutation dans un site donneur d'épissage), l'une par SSCP (single strand conformation polymorphism), l'autre par HMA (heteroduplex mobility analysis).

Dans tous les cas, il en résulte une protéine tronquée et les auteurs concluent à un mécanisme par haplo-insuffisance. Cette hypothèse semble en effet la plus probable, ne serait ce qu'en raison des $7 \%$ à $8 \%$ de cas porteurs d'une délétion qui ont donc complètement perdu le gène. Mais chez la drosophile, la régulation du signal Notch est aussi sensible à l'excès de copies du gène normal qu'à l'haplo-insuffisance, toute modification quantitative ayant pour conséquence de nombreuses anomalies du développement. Et des formes tronquées de Delta et de Serrate peuvent avoir un effet dominant négatif: les protéines sont sécrétées et freinent l'action de Notch.

Chez l'homme, les importantes variations phénotypiques intrafamiliales, pour une même mutation, font suspecter l'intervention d'autres facteurs encore à découvrir, et il n'est pas impossible que d'autres gènes de la région soient en cause dans les cas de grandes délétions, dans cette maladie qui fut d'abord considérée comme un syndrome de gènes contigus et qui comporte une riche symptomatologie.

La voie Notch jouant un rôle capital dans le développement et dans la différenciation cellulaire [1], l'atteinte prédominante des canaux biliaires dans le syndrome d'Alagille est surprenante et laisse présager un délicat équilibre entre le ligand et son récepteur, ainsi qu'une multiplicité de facteurs intervenant tout au long du développement embryonnaire. Nul doute que des mutations d'autres gènes appartenant à cette voie soient retrouvées prochainement dans des maladies génétiques constitutionnelles ou acquises.

S.G. E.T.L.

1. Schweisguth F, Israël A. Signalisation intercellulaire par le récepteur Notch: conservation de la drosophile aux mammifères. Med Sci 1996; 12: 155-63.

2. Larson C, Lardelli M, White I, Lendhal V. The human NOTCH 1,2 and 3 genes are located at chromosome positions 9q34, 1p13-11, and 19p13.2-p13.1 in regions of neoplasia-associated translocation. Genomics 1994; 24: 253-8.

3. Tournier-Lasserve E, Iba-Zizen MT, Romero N, Bousser MG. Autosomal dominant syndrome with stroke like episodes and leukoencephalopathy. Stroke 1991; 22: 1297-302.

4. Ruchoux M, Djelloul G, Vandenhaute B, Pruvo JP, Vermersch P, Ley D. Systemic vascular smooth muscle cell impairement in cerebral autosomal dominant arteriopathy with subcortical infarcts and leucoencephalopathy. Acta Neuropathol 1995; 89: 500-12.

5. Tournier-Lasserve E, Joutel A, Melki J, Weissenbach J, Lathrop M, Chabriat H, Mas JL, et al. Cerebral autosomal dominant arteriopathy with subcortical infarcts and leukoencephalopathy (CADASIL) maps on chromosome 19q12. Nat Genet 1993; 3 : 256-9.

6. Joutel A, Corpechot C, Ducros A, Vahedi K, Chabriat $\mathrm{H}$, et al. Notch 3 mutations in CADASIL, a hereditary adult-onset condition causing stroke and dementia. Nature 1996; 383: 707-10.

7. Byrnes SLB, Harrod MJE, Friedman JM, Howard-Peebles PN. Del (20p) with manifestations of arteriopathic dysplasia. Am J Med Genet 1986; $24: 673-8$.

8. Oda T, Elkahloun AG, Pike BL, Okajima K, Krantz ID, Genin A, et al. Mutations in the human Jagged1 gene are responsible for Alagille syndrome. Nat Genet 1997; 16 : 235-42.

9. Deleuze JF, Hazan J, Dhorne S, Weissenbach J, Hadchouel M. Mapping of microsatellite markers in the Alagille region and screening of microdeletions by genotyping 23 patients. Eur J Hum Genet 1994; $2: 185-90$.

10. Li L, Krantz ID, Deng Y, Genin A, Banta AB, Collins CC, Qi M, Trask BJ, et al. Alagille syndrome is caused by mutations in human Jagged1, which encodes a ligand for Notch1. Nat Genet 1997; 16: 243-51.

\section{Cours de Biologie Moléculaire de la Cellule Enseignement Pratique}

\section{9 mars-10 avril 1998}

Responsables du cours : A. Dautry-Varsat et D. Louvard

Renseignements et Inscriptions, date limite le 1er décembre $1997-$ Mme Banisso

Secrétariat des Enseignements et des Stages

Institut Pasteur, 28, rue du Docteur-Roux, 75724 Paris Cedex 15, France

Tél. : 0145688141 ou 0140613362 -Fax: 0140613046 